\title{
On Common Random Fixed Points of a New Iteration with Errors for Nonself Asymptotically Quasi-Nonexpansive Type Random Mappings
}

\author{
R. A. Rashwan, ${ }^{1}$ P. K. Jhade, ${ }^{2}$ and Dhekra Mohammed Al-Baqeri ${ }^{1}$ \\ ${ }^{1}$ Department of Mathematics, University of Assiut, Assiut 71516, Egypt \\ ${ }^{2}$ Department of Mathematics, NRI Institute of Information Science \& Technology, Bhopal, Madhya Pradesh 462021, India
}

Correspondence should be addressed to P. K. Jhade; pmathsjhade@gmail.com

Received 1 November 2012; Accepted 11 March 2013

Academic Editor: Stefan Kunis

Copyright (c) 2013 R. A. Rashwan et al. This is an open access article distributed under the Creative Commons Attribution License, which permits unrestricted use, distribution, and reproduction in any medium, provided the original work is properly cited.

We prove some strong convergence of a new random iterative scheme with errors to common random fixed points for three and then $N$ nonself asymptotically quasi-nonexpansive-type random mappings in a real separable Banach space. Our results extend and improve the recent results in Kiziltunc, 2011, Thianwan, 2008, Deng et al., 2012, and Zhou and Wang, 2007 as well as many others.

\section{Introduction and Preliminaries}

The theory of random operators is an important branch of probabilistic analysis which plays a key role in many applied areas. The study of random fixed points forms a central topic in this area. Research of this direction was initiated by Prague School of Probabilistic in connection with random operator theory [1-3]. Random fixed point theory has attracted much attention in recent times since the publication of the survey article by Bharucha-Reid [4] in 1976, in which the stochastic versions of some well-known fixed point theorems were proved.

A lot of efforts have been devoted to random fixed point theory and applications (e.g. see [5-10] and many others).

Let $(\Omega, \Sigma)$ be a measurable space, $C$ a nonempty subset of a separable Banach space $E$. A mapping $\xi: \Omega \rightarrow C$ is called measurable if $\xi^{-1}(B \cap C) \in \Sigma$ for every Borel subset $B$ of $E$.

A mapping $T: \Omega \times C \rightarrow C$ is said to be random mapping if for each fixed $x \in C$, the mapping $T(\cdot, x): \Omega \rightarrow C$ is measurable.

A measurable mapping $\xi: \Omega \rightarrow C$ is called a random fixed point of the random mapping $T: \Omega \times C \rightarrow C$ if $T(w, \xi(w))=\xi(w)$ for each $w \in \Omega$.

Throughout this paper, we denote the set of all random fixed points of random mapping $T$ by $R F(T)$ and by $T^{n}(w, x)$ for the $n$th iterate $T(w, T(, \ldots T(w, x)))$ of $T$.
The class of asymptotically nonexpansive mappings is a natural generalization of the important class of nonexpansive mappings. Goebel and Kirk [11] proved that if $C$ is a nonempty closed and bounded subset of a uniformly convex Banach space, then every asymptotically nonexpansive self-mapping has a fixed point.

Iterative techniques for asymptotically nonexpansive selfmappings in Banach spaces including Mann type and Ishikawa type iteration processes have been studied extensively by various authors (e.g. see [12-15]).

The strong and weak convergences of the sequence of Mann iterates to a fixed point of quasi-nonexpansive mappings were studied by Petryshyn and Williamson [16]. Subsequently, the convergence of Ishikawa iterates of quasinonexpansive mappings in Banach spaces were discussed by Ghosh and Debnath [17]. The previous results and some obtained necessary and sufficient conditions for Ishikawa iterative sequence to converge a fixed point for asymptotically quasi-nonexpansive mappings were extended by Liu $[18,19]$.

In 2000, Noor [20] introduced a three-step iterative scheme and studied the approximate solutions of variational inclusion in Hilbert spaces. $\mathrm{Xu}$ and Noor [21] introduced and studied a three-step iterative scheme for asymptotically nonexpansive mappings, and they proved weak and strong convergences theorems for asymptotically nonexpansive mappings in Banach spaces. In 2005, Suantai [22] defined 
a new three-step iteration, which is an extension of Noor iterations, and gave some weak and strong convergences theorems of such iterations for asymptotically nonexpansive mappings in uniformly convex Banach spaces.

For nonself nonexpansive mappings, some authors (e.g., see [23-27]) have studied the strong and weak convergences theorems in Hilbert space or uniformly convex Banach spaces.

A subset $C$ of $E$ is said to be a retract of $E$ if there exists a continuous map $P: E \rightarrow C$ such that $P x=x$ for all $x \in C$. Every closed convex subset of uniformly convex Banach space is a retract. A map $P: E \rightarrow E$ is a retraction if $P^{2}=P$. It follows that if a map $P$ is a retraction, then $P y=y$ for all $y$ in the range of $P$.

The concept of nonself asymptotically nonexpansive mappings was introduced by Chidume et al. [28] in 2003 as the generalization of asymptotically nonexpansive selfmappings.

They studied the following iteration process:

$$
x_{1} \in C, \quad x_{n+1}=P\left(\left(1-\alpha_{n}\right) x_{n}+\alpha_{n} T(P T)^{n-1} x_{n}\right),
$$

where $T: C \rightarrow E$ is an asymptotically nonexpansive nonself mapping, $\left\{\alpha_{n}\right\}$ is a real sequence in $(0,1)$, and $P$ is a nonexpansive retraction from $E$ to $C$.

Wang [29] generalized the result of Chidume et al. [28] and got some new results. He defined and studied the following iteration process:

$$
\begin{gathered}
x_{n+1}=P\left(\left(1-\alpha_{n}\right) x_{n}+\alpha_{n} T_{1}\left(P T_{1}\right)^{n-1} y_{n}\right), \\
y_{n}=P\left(\left(1-\beta_{n}\right) x_{n}+\beta_{n} T_{2}\left(P T_{2}\right)^{n-1} x_{n}\right), \quad x_{1} \in C, n \geq 1,
\end{gathered}
$$

where $T_{1}, T_{2}: C \rightarrow E$ are asymptotically nonexpansive nonself mappings and $\left\{\alpha_{n}\right\},\left\{\beta_{n}\right\}$ are real sequences in $[0,1)$.

Now, we introduce the following concepts for nonself mappings

Definition 1 (see $[28,30,31]$ ). Let $C$ be a nonempty subset of a real separable Banach space and $T: \Omega \times C \rightarrow E$ a nonself random mapping. Then, $T$ is said to be

(1) nonexpansive random operator if for arbitrary $x, y \in$ $C,\|T(w, x)-T(w, y)\| \leq\|x-y\|$, for all $w \in \Omega$

(2) nonself asymptotically nonexpansive random mapping if there exists a sequence of measurable functions $r_{n}(w): \Omega \rightarrow[1, \infty)$ with $\lim _{n \rightarrow \infty} r_{n}(w)=1$ for each $w \in \Omega$ such that for arbitrary $x, y \in C$,

$$
\begin{aligned}
& \left\|T(P T)^{n-1}(w, x)-T(P T)^{n-1}(w, y)\right\| \\
& \quad \leq r_{n}(w)\|x-y\|, \quad \forall w \in \Omega, n \geq 1 ;
\end{aligned}
$$

(3) nonself asymptotically quasi-nonexpansive random mapping if $R F(T) \neq \phi$ and there exists a sequence of measurable functions $r_{n}(w): \Omega \rightarrow[1, \infty)$ with $\lim _{n \rightarrow \infty} r_{n}(w)=1$ for each $w \in \Omega$ such that

$$
\begin{aligned}
& \left\|T(P T)^{n-1}(w, \eta(w))-\xi(w)\right\| \\
& \quad \leq r_{n}(w)\|\eta(w)-\xi(w)\|, \quad \forall w \in \Omega, n \geq 1,
\end{aligned}
$$

where $\xi(w): \Omega \rightarrow C$ is a random fixed point of $T$ and $\eta(w): \Omega \rightarrow C$ is any measurable mapping;

(4) nonself asymptotically nonexpansive-type random mapping if

$$
\begin{array}{r}
\limsup _{n \rightarrow \infty}\left\{\operatorname { s u p } _ { x , y \in C } \left\{\left\|T(P T)^{n-1}(w, x)-T(P T)^{n-1}(w, y)\right\|^{2}\right.\right. \\
\left.\left.-\|x-y\|^{2}\right\}\right\} \leq 0, \quad \forall w \in \Omega, n \geq 1 ;
\end{array}
$$

(5) Nonself asymptotically quasi-nonexpansive-type random mapping if $R F(T) \neq \phi$, and

$$
\begin{array}{r}
\limsup _{n \rightarrow \infty}\left\{\operatorname { s u p } _ { \xi ( w ) \in F } \left\{\left\|T(P T)^{n-1}(w, \eta(w))-\xi(w)\right\|^{2}\right.\right. \\
\left.\left.-\|\eta(w)-\xi(w)\|^{2}\right\}\right\} \leq 0, \\
\forall w \in \Omega, n \geq 1,
\end{array}
$$

where $\xi(w): \Omega \rightarrow C$ is a random fixed point of $T$ and $\eta(w): \Omega \rightarrow C$ is any measurable mapping.

Remark 2. (1) If $T: \Omega \times C \rightarrow E$ is a nonself asymptotically nonexpansive random mapping, then $T$ is a nonself asymptotically nonexpansive-type random mapping.

(2) If $R F(T) \neq \phi$ and $T: \Omega \times C \rightarrow E$ is a nonself asymptotically quasi-nonexpansive random mapping, then $T$ is a nonself asymptotically quasi-nonexpansive-type random mapping.

(3) If $R F(T) \neq \phi$ and $T: \Omega \times C \rightarrow E$ is a nonself asymptotically nonexpansive-type random mapping, then $T$ is a nonself asymptotically quasi-nonexpansive-type random mapping.

Remark 3. Observe that for any measurable mapping $\eta(w)$ : $\Omega \rightarrow C$ and $\xi(w) \in F$, we have

$$
\begin{gathered}
\limsup _{n \rightarrow \infty}\left\{\operatorname { s u p } _ { \xi ( w ) \in F } \left\{\left\|T(P T)^{n-1}(w, \eta(w))-\xi(w)\right\|^{2}\right.\right. \\
\left.\left.-\|\eta(w)-\xi(w)\|^{2}\right\}\right\} \leq 0,
\end{gathered}
$$

which implies

$$
\begin{aligned}
\limsup _{n \rightarrow \infty}\left\{\sup _{\xi(w) \in F}\{(\right. & \left\|T(P T)^{n-1}(w, \eta(w))-\xi(w)\right\| \\
& -\|\eta(w)-\xi(w)\|) \\
& \times\left(\left\|T(P T)^{n-1}(w, \eta(w))-\xi(w)\right\|\right. \\
& +\|\eta(w)-\xi(w)\|)\}\} \leq 0 .
\end{aligned}
$$


Therefore,

$$
\begin{gathered}
\limsup _{n \rightarrow \infty}\left\{\operatorname { s u p } _ { \xi ( w ) \in F } \left\{\left(\left\|T(P T)^{n-1}(w, \eta(w))-\xi(w)\right\|\right.\right.\right. \\
-\|\eta(w)-\xi(w)\|)\}\} \leq 0 .
\end{gathered}
$$

In [25], Shahzad studied the following iterative sequences:

$$
\begin{array}{r}
x_{n+1}=P\left(\left(1-\alpha_{n}\right) x_{n}+\alpha_{n} T P\left[\left(1-\beta_{n}\right) x_{n}+\beta_{n} T x_{n}\right]\right), \\
x_{1} \in C, \quad n \geq 1,
\end{array}
$$

where $T: C \rightarrow E$ is a nonexpansive nonself mapping, $C$ is a nonempty closed convex nonexpansive retract of a real uniformly convex Banach space $E$ with $P$ being a nonexpansive retraction from $E$ to $C$, and $\left\{\alpha_{n}\right\},\left\{\beta_{n}\right\}$ are real sequences in $[0,1)$.

Recently, Thianwan [32] generalized the iteration process (10) as follows:

$$
\begin{aligned}
x_{n+1}=P & \left(\left(1-\alpha_{n}-\gamma_{n}\right) x_{n}\right. \\
& \left.+\alpha_{n} T P\left[\left(1-\beta_{n}\right) y_{n}+\beta_{n} T y_{n}\right]+\gamma_{n} u_{n}\right), \\
y_{n}=P( & \left(1-\alpha_{n}^{\prime}-\gamma_{n}^{\prime}\right) x_{n}+\alpha_{n}^{\prime} T P \\
& \left.\times\left[\left(1-\beta_{n}^{\prime}\right) x_{n}+\beta_{n}^{\prime} T x_{n}\right]+\gamma_{n}^{\prime} v_{n}\right), \quad x_{1} \in C, n \geq 1,
\end{aligned}
$$

where $\left\{\alpha_{n}\right\},\left\{\beta_{n}\right\},\left\{\gamma_{n}\right\},\left\{\alpha_{n}^{\prime}\right\},\left\{\beta_{n}^{\prime}\right\},\left\{\gamma_{n}^{\prime}\right\}$ are appropriate sequences in $[0,1)$ and $\left\{u_{n}\right\},\left\{v_{n}\right\}$ are bounded sequences in $C$. He proved weak and strong convergences theorems for nonexpansive nonself mappings in uniformly convex Banach spaces.

In 2011, Kiziltunc [33] studied the strong convergence to a common fixed point of a new iterative scheme for two nonself asymptotically quasi-nonexpansive-type mappings in Banach spaces defined as follows:

$$
\begin{aligned}
& x_{n+1}=P(\left(1-a_{n}\right) x_{n}+a_{n} S(P S)^{n-1} \\
&\left.\times\left(\left(1-\alpha_{n}\right) y_{n}+\alpha_{n} S(P S)^{n-1} y_{n}\right)\right), \\
& y_{n}=P(\left(1-b_{n}\right) x_{n}+b_{n} T(P T)^{n-1} \\
& \times\left.\left(\left(1-\beta_{n}\right) x_{n}+\beta_{n} T(P T)^{n-1} x_{n}\right)\right), \\
& x_{1} \in C, n \geq 1,
\end{aligned}
$$

where $\left\{a_{n}\right\},\left\{b_{n}\right\},\left\{\alpha_{n}\right\},\left\{\beta_{n}\right\}$ are appropriate sequences in $[0,1)$.

More recently, Deng et al. [34] obtained the strong and weak convergences theorems for common fixed points of two nonself asymptotically nonexpansive mappings in Banach spaces.
The iterative scheme is defined as follows:

$$
\begin{gathered}
x_{n+1}=\alpha_{n 1} x_{n}+\beta_{n 1}\left(P T_{1}\right)^{n} y_{n}+\gamma_{n 1}\left(P T_{2}\right)^{n} y_{n}, \\
y_{n}=\alpha_{n 2} x_{n}+\beta_{n 2}\left(P T_{1}\right)^{n} z_{n}+\gamma_{n 2}\left(P T_{2}\right)^{n} z_{n}, \\
z_{n}=\alpha_{n 3} x_{n}+\beta_{n 3}\left(P T_{1}\right)^{n} x_{n}+\gamma_{n 3}\left(P T_{2}\right)^{n} x_{n},
\end{gathered}
$$

where $\left\{\alpha_{n i}\right\},\left\{\beta_{n i}\right\},\left\{\gamma_{n i}\right\} \quad(i=1,2,3)$ are appropriate sequences in $[a, 1-a]$ for some $a \in(0,1)$ satisfying $\alpha_{n i}+\beta_{n i}+\gamma_{n i}=$ $1 \quad(i=1,2,3)$.

For random operators, Beg and Abbas [30] studied the different random iterative algorithms for weakly contractive and asymptotically nonexpansive random operators on arbitrary Banach space. They also established convergence of an implicit random iterative process to a common fixed point for a finite family of asymptotically quasi-nonexpansive operators. Plubtieng et al. [35, 36] studied weak and strong convergences theorems for a modified random Noor iterative scheme with errors for three asymptotically nonexpansive self-mappings in Banach space defined as follows:

$$
\begin{array}{r}
\xi_{n+1}(w)=\alpha_{n} T_{1}^{n}\left(w, \eta_{n}(w)\right)+\beta_{n} \xi_{n}(w)+\gamma_{n} f_{n}(w), \\
\eta_{n}(w)=\alpha_{n}^{\prime} T_{2}^{n}\left(w, \zeta_{n}(w)\right)+\beta_{n}^{\prime} \xi_{n}(w)+\gamma_{n}^{\prime} f_{n}^{\prime}(w), \\
\zeta_{n}(w)=\alpha_{n}^{\prime \prime} T_{3}^{n}\left(w, \xi_{n}(w)\right)+\beta_{n}^{\prime \prime} \xi_{n}(w)+\gamma_{n}^{\prime \prime} f_{n}^{\prime \prime}(w), \\
n \geq 1, w \in \Omega,
\end{array}
$$

where $T_{1}, T_{2}, T_{3}: \Omega \times C \rightarrow C$ are three asymptotically nonexpansive random self-mappings, $\xi_{1}: \Omega \rightarrow C$ is an arbitrary given measurable mapping from $\Omega$ to $C,\left\{f_{n}(w)\right\}$, $\left\{f_{n}^{\prime}(w)\right\},\left\{f_{n}^{\prime \prime}(w)\right\}$ are bounded sequence of measurable functions from $\Omega$ to $C$, and $\left\{\alpha_{n}\right\},\left\{\alpha_{n}^{\prime}\right\},\left\{\alpha_{n}^{\prime \prime}\right\},\left\{\beta_{n}\right\},\left\{\beta_{n}^{\prime}\right\},\left\{\beta_{n}^{\prime \prime}\right\}$, $\left\{\gamma_{n}\right\},\left\{\gamma_{n}^{\prime}\right\},\left\{\gamma_{n}^{\prime \prime}\right\}$ are sequences of real numbers in $[0,1]$ with $\alpha_{n}+\beta_{n}+\gamma_{n}=\alpha_{n}^{\prime}+\beta_{n}^{\prime}+\gamma_{n}^{\prime}=\alpha_{n}^{\prime \prime}+\beta_{n}^{\prime \prime}+\gamma_{n}^{\prime \prime}=1$.

Remark 4. If $T_{1}=T_{2}=T_{3}=T$ and $\gamma_{n}=\gamma_{n}^{\prime}=\gamma_{n}^{\prime \prime}=0$, then (14) becomes as follows:

$$
\begin{aligned}
\xi_{n+1}(w) & =\alpha_{n} T^{n}\left(w, \eta_{n}(w)\right)+\beta_{n} \xi_{n}(w), \\
\eta_{n}(w) & =\alpha_{n}^{\prime} T^{n}\left(w, \zeta_{n}(w)\right)+\beta_{n}^{\prime} \xi_{n}(w), \\
\zeta_{n}(w) & =\alpha_{n}^{\prime \prime} T_{3}^{n}\left(w, \xi_{n}(w)\right)+\beta_{n}^{\prime \prime} \xi_{n}(w), \quad n \geq 1, w \in \Omega,
\end{aligned}
$$

which was studied by Beg and Abbas in [30].

For nonself random mappings, Zhou and Wang [37] studied the approximation of the following iteration process:

$$
\begin{gathered}
\xi_{n+1}(w)=P\left(\left(1-\alpha_{n}\right) \xi_{n}(w)\right. \\
\left.+\alpha_{n} T(P T)^{n-1}\left(w, \eta_{n}(w)\right)\right), \\
\eta_{n}(w)=P\left(\left(1-\beta_{n}\right) \xi_{n}(w)+\beta_{n} T(P T)^{n-1}\left(w, \xi_{n}(w)\right)\right), \\
n \geq 1, w \in \Omega,
\end{gathered}
$$


where $T: \Omega \times C \rightarrow E$ is an asymptotically nonexpansive nonself random mapping, $\xi_{1}: \Omega \rightarrow C$ is an arbitrary given measurable mapping from $\Omega$ to $C,\left\{\alpha_{n}\right\},\left\{\beta_{n}\right\}$ are sequences in $[0,1]$, and $P$ is a nonexpansive retraction from $E$ to $C$.

Saluja [38] and many other authors extended the results of Zhou and Wang [37] by studying multistep random iteration scheme with errors for common random fixed point of a finite family of nonself asymptotically nonexpansive random mapping in real uniformly separable Banach spaces.

Inspired and motivated by [32-34, 37] and others, we introduced a new three-step and $N$-step random iterative scheme with errors for asymptotically quasi-nonexpansivetype nonself random mappings in a separable Banach space. Some strong convergences theorems are established for these new random iterative schemes with errors in separable Banach space. The iterative scheme for three nonself random mappings is defined as follows.

Definition 5. Let $T_{1}, T_{2}, T_{3}: \Omega \times C \rightarrow C$ be three nonself random mappings, where $C$ is a nonempty closed convex subset of a separable Banach space $E$, and $P: E \rightarrow C$ is a nonexpansive retraction of $E$ onto $C$. Let $\xi_{1}(w): \Omega \rightarrow C$ be a measurable mapping. Suppose that $\left\{\xi_{n}(w)\right\}$ is generated iteratively by $\xi_{1}(w) \in C$, having

$$
\begin{aligned}
& \xi_{n+1}(w)=P\left[\left(1-a_{n}-\sigma_{n}\right) \xi_{n}(w)+a_{n} T_{1}\left(P T_{1}\right)^{n-1}\right. \\
& \times\left(w,\left(1-\alpha_{n}\right) \eta_{n}(w)\right. \\
&\left.\left.+\alpha_{n} T_{1}\left(P T_{1}\right)^{n-1}\left(w, \eta_{n}(w)\right)\right)+\sigma_{n} f_{n}(w)\right], \\
& \eta_{n}(w)=P\left[\left(1-b_{n}-\delta_{n}\right) \xi_{n}(w)+b_{n} T_{2}\left(P T_{2}\right)^{n-1}\right. \times\left(w,\left(1-\beta_{n}\right) \zeta_{n}(w)\right. \\
&\left.\left.\quad+\beta_{n} T_{2}\left(P T_{2}\right)^{n-1}\left(w, \zeta_{n}(w)\right)\right)+\delta_{n} g_{n}(w)\right], \\
& \zeta_{n}(w)=P\left[\left(1-c_{n}-\lambda_{n}\right) \xi_{n}(w)+c_{n} T_{3}\left(P T_{3}\right)^{n-1}\right. \\
& \times\left(w,\left(1-\gamma_{n}\right) \xi_{n}(w)\right. \\
&\left.\left.\quad+\gamma_{n} T_{3}\left(P T_{3}\right)^{n-1}\left(w, \xi_{n}(w)\right)\right)+\lambda_{n} h_{n}(w)\right],
\end{aligned}
$$

for all $n \geq 1, w \in \Omega$, where $\left\{a_{n}\right\},\left\{b_{n}\right\},\left\{c_{n}\right\},\left\{\alpha_{n}\right\},\left\{\beta_{n}\right\},\left\{\gamma_{n}\right\}$, $\left\{\sigma_{n}\right\},\left\{\delta_{n}\right\}$, and $\left\{\lambda_{n}\right\}$ are sequences in $[0,1]$ such that $a_{n}+\sigma_{n} \leq$ $1, b_{n}+\delta_{n} \leq 1, c_{n}+\lambda_{n} \leq 1$, and $\left\{f_{n}(w)\right\},\left\{g_{n}(w)\right\},\left\{h_{n}(w)\right\}$ are bounded sequences of measurable functions from $\Omega$ to $C$ for all $w \in \Omega$.

Definition 5 can be extended to $N$ nonself random mappings as follows.

Definition 6. Let $T_{1}, T_{2}, \ldots, T_{N}: \Omega \times C \rightarrow C$ be $N$ nonself random mappings, where $C$ is a nonempty closed convex subset of a separable Banach space $E$, and $P: E \rightarrow C$ is a nonexpansive retraction of $E$ onto $C$. Let $\xi_{1}(w): \Omega \rightarrow C$ be a measurable mapping. Define sequences function $\left\{\xi_{n}^{(N)}(w)\right\}$, $\left\{\xi_{n}^{(N-1)}(w)\right\}, \ldots,\left\{\xi_{n}^{(1)}(w)\right\}$ in $C$ as follows:

$$
\begin{aligned}
& \xi_{n+1}(w)=\xi_{n}^{(N)}(w) \\
& =P\left[\left(1-a_{n}^{(N)}-\sigma_{n}^{(N)}\right) \xi_{n}(w)+a_{n}^{(N)} T_{N}\left(P T_{N}\right)^{n-1}\right. \\
& \times\left(w,\left(1-\alpha_{n}^{(N)}\right) \xi_{n}^{(N-1)}(w)+\alpha_{n}^{(N)} T_{N}\right. \\
& \left.\left.\times\left(P T_{N}\right)^{n-1}\left(w, \xi_{n}^{(N-1)}(w)\right)\right)+\sigma_{n}^{(N)} f_{n}^{(N)}\right], \\
& \xi_{n}^{(N-1)}(w)=P\left[\left(1-a_{n}^{(N-1)}-\sigma_{n}^{(N-1)}\right) \xi_{n}(w)\right. \\
& +a_{n}^{(N-1)} T_{N-1}\left(P T_{N-1}\right)^{n-1} \\
& \times\left(w,\left(1-\alpha_{n}^{(N-1)}\right) \xi_{n}^{(N-2)}(w)\right. \\
& \left.+\alpha_{n}^{(N-1)} T_{N-1}\left(P T_{N-1}\right)^{n-1}\left(w, \xi_{n}^{(N-2)}(w)\right)\right) \\
& \left.+\sigma_{n}^{(N-1)} f_{n}^{(N-1)}\right] \text {, } \\
& \cdots=\cdots \\
& \ldots=\ldots \\
& \xi_{n}^{(1)}(w)=P\left[\left(1-a_{n}^{(1)}-\sigma_{n}^{(1)}\right) \xi_{n}(w)+a_{n}^{(1)} T_{1}\left(P T_{1}\right)^{n-1}\right. \\
& \times\left(w,\left(1-\alpha_{n}^{(1)}\right) \xi_{n}(w)+\alpha_{n}^{(1)} T_{1}\left(P T_{1}\right)^{n-1}\right. \\
& \left.\left.\times\left(w, \xi_{n}(w)\right)\right)+\sigma_{n}^{(1)} f_{n}^{(1)}\right], \\
& n \geq 1, w \in \Omega \text {, }
\end{aligned}
$$

where $\left\{a_{n}^{(i)}\right\},\left\{\alpha_{n}^{(i)}\right\}$, and $\left\{\sigma_{n}^{(i)}\right\}(i=1,2, \ldots, N)$ are sequences in $[0,1]$ such that $a_{n}^{(i)}+\sigma_{n}^{(i)} \leq 1$, for all $(i=1,2, \ldots, N)$, and $\left\{f_{n}^{(i)}\right\} \quad(i=1,2, \ldots, N)$ are bounded sequences of measurable functions from $\Omega$ to $C$ for all $w \in \Omega$.

The following lemma is useful for proving our results.

Lemma 7 (see [39]). Let $\left\{a_{n}\right\},\left\{b_{n}\right\}$ and $\left\{m_{n}\right\}$ be nonnegative real sequences satisfying

$$
a_{n+1} \leq\left(1+m_{n}\right) a_{n}+b_{n}, \quad n \geq 1 .
$$

If $\sum_{n=1}^{\infty} m_{n}<\infty$ and $\sum_{n=1}^{\infty} b_{n}<\infty$, then

(1) $\lim _{n \rightarrow \infty} a_{n}$ exists;

(2) $\lim _{n \rightarrow \infty} a_{n}=0$ whenever $\liminf _{n \rightarrow \infty} a_{n}=0$.

\section{Main Results}

In this section, we will first prove the strong convergence of the iterative scheme (17) to a common random fixed point for three asymptotically quasi-nonexpansive-type nonself random mappings in a separable Banach space. Then, we extend the obtained results to $N$ asymptotically quasi-nonexpansivetype nonself random mappings by using the iterative scheme (18). Finally, we use Theorem 8 and Condition (A) [40] to obtain a convergences theorem for scheme (17). 
Theorem 8. Let $E$ be a real separable Banach space and $C$ a nonempty closed convex subset of $E$ with $P$ being a nonexpansive retraction. Let $T_{i}: \Omega \times C \rightarrow E, i=1,2,3$, be three asymptotically quasi-nonexpansive-type nonself random mappings with $F=\bigcap_{i=1}^{3} R F\left(T_{i}\right) \neq \phi$, for all $w \in \Omega$. Suppose that $\left\{\xi_{n}(w)\right\},\left\{\eta_{n}(w)\right\}$ and $\left\{\zeta_{n}(w)\right\}$ are the sequences defined as in (17) where $\left\{a_{n}\right\},\left\{b_{n}\right\},\left\{c_{n}\right\},\left\{\alpha_{n}\right\},\left\{\beta_{n}\right\},\left\{\gamma_{n}\right\},\left\{\sigma_{n}\right\},\left\{\delta_{n}\right\}$, and $\left\{\lambda_{n}\right\}$ are sequences in $[0,1]$ such that $a_{n}+\sigma_{n} \leq 1, b_{n}+$ $\delta_{n} \leq 1, c_{n}+\lambda_{n} \leq 1$ and $\left\{f_{n}(w)\right\},\left\{g_{n}(w)\right\},\left\{h_{n}(w)\right\}$ are bounded sequences of measurable functions from $\Omega$ to $C$ with the following restrictions: $\sum_{n=1}^{\infty} \sigma_{n}<\infty, \sum_{n=1}^{\infty} \delta_{n}<\infty$, and $\sum_{n=1}^{\infty} \lambda_{n}<\infty$. Then, $\left\{\xi_{n}(w)\right\}$ converge to a common random fixed point of $T_{1}, T_{2}$, and $T_{3}$ if and only if

$$
\liminf _{n \rightarrow \infty} d\left(\xi_{n}(w), F\right)=0, \quad w \in \Omega
$$

Proof. The necessity of (20) is obvious.

Next, we prove the sufficiency of (20). Let $\xi(w) \in$ $F=\bigcap_{i=1}^{3} R F\left(T_{i}\right)$; by the boundedness of the sequences of measurable functions $\left\{f_{n}(w)\right\},\left\{g_{n}(w)\right\},\left\{h_{n}(w)\right\}$, we put for each $w \in \Omega$,

$$
\begin{aligned}
M(w)=\max \{ & \sup _{n \geq 1, \xi \in F}\left\|f_{n}(w)-\xi(w)\right\| \vee \\
& \times \sup _{n \geq 1, \xi \in F}\left\|g_{n}(w)-\xi(w)\right\| \vee \\
& \left.\times \sup _{n \geq 1, \xi \in F}\left\|h_{n}(w)-\xi(w)\right\|\right\} .
\end{aligned}
$$

Then, $M(w)<\infty$ for each $w \in \Omega$.

Since $\xi(w) \in F$ and $\eta(w): \Omega \rightarrow C$ is any measurable mapping, we have

$$
\begin{aligned}
\limsup _{n \rightarrow \infty}\left\{\operatorname { s u p } _ { \xi ( w ) \in F } \left\{\left\|T_{i}\left(P T_{i}\right)^{n-1}(w, \eta(w))-\xi(w)\right\|\right.\right. \\
-\|\eta(w)-\xi(w)\|\}\} \leq 0, \quad i=1,2,3 .
\end{aligned}
$$

It follows that for any given $\epsilon>0$, there exists a positive integer $n_{0}$ such that for $n \geq n_{0}$ and $\xi(w) \in F$, we have

$$
\begin{aligned}
\sup _{\xi(w) \in F}\left\{\left\|T_{i}\left(P T_{i}\right)^{n-1}(w, \eta(w))-\xi(w)\right\|\right. \\
-\|\eta(w)-\xi(w)\|\} \leq \epsilon, \quad i=1,2,3 .
\end{aligned}
$$

Since $\left\{\xi_{n}(w)\right\},\left\{\eta_{n}(w)\right\}$, and $\left\{\zeta_{n}(w)\right\} \subset E$, then we have for $w \in \Omega$,

$$
\begin{array}{r}
\left\{\left\|T_{1}\left(P T_{1}\right)^{n-1}\left(w, \eta_{n}(w)\right)-\xi(w)\right\|-\left\|\eta_{n}(w)-\xi(w)\right\|\right\} \leq \epsilon, \\
\forall n \geq n_{0}, \quad \forall \xi \in F,
\end{array}
$$

$$
\begin{array}{r}
\left\{\left\|T_{2}\left(P T_{2}\right)^{n-1}\left(w, \zeta_{n}(w)\right)-\xi(w)\right\|-\left\|\zeta_{n}(w)-\xi(w)\right\|\right\} \leq \epsilon, \\
\forall n \geq n_{0}, \quad \forall \xi \in F,
\end{array}
$$

$$
\begin{array}{r}
\left\{\left\|T_{3}\left(P T_{3}\right)^{n-1}\left(w, \xi_{n}(w)\right)-\xi(w)\right\|-\left\|\xi_{n}(w)-\xi(w)\right\|\right\} \leq \epsilon, \\
\forall n \geq n_{0}, \forall \xi \in F .
\end{array}
$$

Setting for $w \in \Omega$,

$$
\begin{gathered}
\mu_{n}(w)=\left(1-\alpha_{n}\right) \eta_{n}(w)+\alpha_{n} T_{1}\left(P T_{1}\right)^{n-1}\left(w, \eta_{n}(w)\right), \\
v_{n}(w)=\left(1-\beta_{n}\right) \zeta_{n}(w)+\beta_{n} T_{2}\left(P T_{2}\right)^{n-1}\left(w, \zeta_{n}(w)\right), \\
\tau_{n}(w)=\left(1-\gamma_{n}\right) \xi_{n}(w)+\gamma_{n} T_{3}\left(P T_{3}\right)^{n-1}\left(w, \xi_{n}(w)\right) .
\end{gathered}
$$

Thus, for $\xi(w) \in F$ and $w \in \Omega$, using (17) and (24), we have

$$
\begin{aligned}
& \left\|\xi_{n+1}(w)-\xi(w)\right\| \\
& =\| P\left[\left(1-a_{n}-\sigma_{n}\right) \xi_{n}(w)+a_{n} T_{1}\left(P T_{1}\right)^{n-1}\right. \\
& \left.\times\left(w, \mu_{n}(w)\right)+\sigma_{n} f_{n}(w)\right]-\xi(w) \| \\
& \leq \|\left(1-a_{n}-\sigma_{n}\right) \xi_{n}(w)+a_{n} T_{1}\left(P T_{1}\right)^{n-1} \\
& \left(w,\left(1-\alpha_{n}\right) \eta_{n}(w)+\alpha_{n} T_{1}\left(P T_{1}\right)^{n-1}\left(w, \eta_{n}(w)\right)\right) \\
& +\sigma_{n} f_{n}(w)-\xi(w) \| \\
& =\|\left(1-a_{n}-\sigma_{n}\right) \xi_{n}(w)+a_{n} \xi(w)+\sigma_{n} \xi(w)-\xi(w) \\
& +a_{n}\left(T_{1}\left(P T_{1}\right)^{n-1}\left(w, \mu_{n}(w)\right)-\xi(w)\right) \\
& +\sigma_{n}\left(f_{n}(w)-\xi(w)\right) \| \\
& \leq\left(1-a_{n}-\sigma_{n}\right)\left\|\xi_{n}(w)-\xi(w)\right\| \\
& +a_{n}\left\|T_{1}\left(P T_{1}\right)^{n-1}\left(w, \mu_{n}(w)\right)-\xi(w)\right\| \\
& +\sigma_{n}\left\|f_{n}(w)-\xi(w)\right\| \\
& \leq\left(1-a_{n}-\sigma_{n}\right)\left\|\xi_{n}(w)-\xi(w)\right\| \\
& +a_{n}\left[\left\|T_{1}\left(P T_{1}\right)^{n-1}\left(w, \mu_{n}(w)\right)-\xi(w)\right\|\right. \\
& \left.-\left\|\mu_{n}(w)-\xi(w)\right\|\right] \\
& +a_{n}\left\|\mu_{n}(w)-\xi(w)\right\|+\sigma_{n} M(w) \\
& \leq\left(1-a_{n}-\sigma_{n}\right)\left\|\xi_{n}(w)-\xi(w)\right\|+a_{n} \epsilon \\
& +a_{n}\left\|\mu_{n}(w)-\xi(w)\right\|+\sigma_{n} M(w) .
\end{aligned}
$$


In addition, by (24), we obtain

$$
\begin{aligned}
\| \mu_{n}(w) & -\xi(w) \| \\
= & \left\|\left(1-\alpha_{n}\right) \eta_{n}(w)+\alpha_{n} T_{1}\left(P T_{1}\right)^{n-1}\left(w, \eta_{n}(w)\right)-\xi(w)\right\| \\
\leq & \left(1-\alpha_{n}\right)\left\|\eta_{n}(w)-\xi(w)\right\| \\
& +\alpha_{n}\left\|T_{1}\left(P T_{1}\right)^{n-1}\left(w, \eta_{n}(w)\right)-\xi(w)\right\| \\
\leq & \left(1-\alpha_{n}\right)\left\|\eta_{n}(w)-\xi(w)\right\|+\alpha_{n} \epsilon+\alpha_{n}\left\|\eta_{n}(w)-\xi(w)\right\| \\
= & \left\|\eta_{n}(w)-\xi(w)\right\|+\alpha_{n} \epsilon .
\end{aligned}
$$

Again using (17) and (25), we have

$$
\begin{aligned}
\left\|\eta_{n}(w)-\xi(w)\right\| \\
=\| P\left[\left(1-b_{n}-\delta_{n}\right) \xi_{n}(w)+b_{n} T_{2}\left(P T_{2}\right)^{n-1}\left(w, v_{n}(w)\right)\right. \\
\left.\quad+\delta_{n} g_{n}(w)\right]-\xi(w) \| \\
\leq \|\left(1-b_{n}-\delta_{n}\right) \xi_{n}(w)+b_{n} T_{2}\left(P T_{2}\right)^{n-1} \\
\quad \times\left(w,\left(1-\beta_{n}\right) \zeta_{n}(w)+\beta_{n} T_{2}\left(P T_{2}\right)^{n-1}\left(w, \zeta_{n}(w)\right)\right) \\
\quad+\delta_{n} g_{n}(w)-\xi(w) \| \\
=\|\left(1-b_{n}-\delta_{n}\right) \xi_{n}(w)+b_{n} \xi(w)+\delta_{n} \xi(w)-\xi(w) \\
\quad+b_{n}\left(T_{2}\left(P T_{2}\right)^{n-1}\left(w, v_{n}(w)\right)-\xi(w)\right) \\
\quad+\delta_{n}\left(g_{n}(w)-\xi(w)\right) \| \\
\leq\left(1-b_{n}-\delta_{n}\right)\left\|\xi_{n}(w)-\xi(w)\right\| \\
+b_{n}\left\|T_{2}\left(P T_{2}\right)^{n-1}\left(w, v_{n}(w)\right)-\xi(w)\right\| \\
+\delta_{n}\left\|g_{n}(w)-\xi(w)\right\| \\
\leq\left(1-b_{n}-\delta_{n}\right)\left\|\xi_{n}(w)-\xi(w)\right\|+b_{n} \epsilon \\
+b_{n}\left\|v_{n}(w)-\xi(w)\right\|+\delta_{n} M(w) .
\end{aligned}
$$

In addition, by (25), we have

$$
\begin{aligned}
\| v_{n}(w) & -\xi(w) \| \\
= & \left\|\left(1-\beta_{n}\right) \zeta_{n}(w)+\beta_{n} T_{2}\left(P T_{2}\right)^{n-1}\left(w, \zeta_{n}(w)\right)-\xi(w)\right\| \\
\leq & \left(1-\beta_{n}\right)\left\|\zeta_{n}(w)-\xi(w)\right\| \\
& +\beta_{n}\left\|T_{2}\left(P T_{2}\right)^{n-1}\left(w, \zeta_{n}(w)\right)-\xi(w)\right\| \\
\leq & \left(1-\beta_{n}\right)\left\|\zeta_{n}(w)-\xi(w)\right\|+\beta_{n} \epsilon+\beta_{n}\left\|\zeta_{n}(w)-\xi(w)\right\| \\
= & \left\|\zeta_{n}(w)-\xi(w)\right\|+\beta_{n} \epsilon .
\end{aligned}
$$

Also, by (17) and (26), we have

$$
\begin{aligned}
&\left\|\zeta_{n}(w)-\xi(w)\right\| \\
&=\| P\left[\left(1-c_{n}-\lambda_{n}\right) \xi_{n}(w)+c_{n} T_{3}\left(P T_{3}\right)^{n-1}\right. \\
&\left.\quad \times\left(w, \tau_{n}(w)\right)+\lambda_{n} h_{n}(w)\right]-\xi(w) \| \\
& \leq \|\left(1-c_{n}-\lambda_{n}\right) \xi_{n}(w)+c_{n} T_{3}\left(P T_{3}\right)^{n-1} \\
& \quad \times\left(w,\left(1-\gamma_{n}\right) \xi_{n}(w)+\gamma_{n} T_{3}\left(P T_{3}\right)^{n-1}\left(w, \xi_{n}(w)\right)\right) \\
& \quad+\lambda_{n} h_{n}(w)-\xi(w) \| \\
&=\|\left(1-c_{n}-\lambda_{n}\right) \xi_{n}(w)+c_{n} \xi(w)+\lambda_{n} \xi(w)-\xi(w) \\
&+c_{n}\left(T_{3}\left(P T_{3}\right)^{n-1}\left(w, \tau_{n}(w)\right)-\xi(w)\right) \\
&+\lambda_{n}\left(h_{n}(w)-\xi(w)\right) \| \\
& \leq\left(1-c_{n}-\lambda_{n}\right)\left\|\xi_{n}(w)-\xi(w)\right\| \\
&+ c_{n}\left\|T_{3}\left(P T_{3}\right)^{n-1}\left(w, \tau_{n}(w)\right)-\xi(w)\right\| \\
&+ \lambda_{n}\left\|h_{n}(w)-\xi(w)\right\| \\
& \leq\left(1-c_{n}-\lambda_{n}\right)\left\|\xi_{n}(w)-\xi(w)\right\|+c_{n} \epsilon \\
&+ c_{n}\left\|\tau_{n}(w)-\xi(w)\right\|+\lambda_{n} M(w) .
\end{aligned}
$$

In addition, by (26), we have

$$
\begin{aligned}
\| \tau_{n}(w) & -\xi(w) \| \\
= & \left\|\left(1-\gamma_{n}\right) \xi_{n}(w)+\gamma_{n} T_{3}\left(P T_{3}\right)^{n-1}\left(w, \xi_{n}(w)\right)-\xi(w)\right\| \\
\leq & \left(1-\gamma_{n}\right)\left\|\xi_{n}(w)-\xi(w)\right\| \\
& +\gamma_{n}\left\|T_{3}\left(P T_{3}\right)^{n-1}\left(w, \xi_{n}(w)\right)-\xi(w)\right\| \\
\leq & \left(1-\gamma_{n}\right)\left\|\xi_{n}(w)-\xi(w)\right\|+\gamma_{n} \epsilon+\gamma_{n}\left\|\xi_{n}(w)-\xi(w)\right\| \\
= & \left\|\xi_{n}(w)-\xi(w)\right\|+\gamma_{n} \epsilon .
\end{aligned}
$$

Substituting (29), (30), (31), (32), and (33) into (28) and simplifying, we obtain

$$
\begin{aligned}
&\left\|\xi_{n+1}(w)-\xi(w)\right\| \\
& \leq\left(1-a_{n}-\sigma_{n}\right)\left\|\xi_{n}(w)-\xi(w)\right\|+a_{n} \epsilon \\
&+a_{n}\left\|\mu_{n}(w)-\xi(w)\right\|+\sigma_{n} M(w) \\
& \leq\left(1-a_{n}-\sigma_{n}\right)\left\|\xi_{n}(w)-\xi(w)\right\|+a_{n} \epsilon \\
&+a_{n}\left[\left\|\eta_{n}(w)-\xi(w)\right\|+\alpha_{n} \epsilon\right]+\sigma_{n} M(w) \\
&=\left(1-a_{n}-\sigma_{n}\right)\left\|\xi_{n}(w)-\xi(w)\right\|+a_{n} \epsilon \\
&+a_{n}\left\|\eta_{n}(w)-\xi(w)\right\|+a_{n} \alpha_{n} \epsilon+\sigma_{n} M(w)
\end{aligned}
$$




$$
\begin{array}{lll}
\leq\left(1-a_{n}-\sigma_{n}\right)\left\|\xi_{n}(w)-\xi(w)\right\|+a_{n} \epsilon & \left(1-\sigma_{n}-a_{n} \delta_{n}-a_{n} b_{n} \lambda_{n}\right)\left\|\xi_{n}(w)-\xi(w)\right\| \\
+a_{n}\left[\left(1-b_{n}-\delta_{n}\right)\left\|\xi_{n}(w)-\xi(w)\right\|+b_{n} \epsilon\right. & & +a_{n} \epsilon+a_{n} b_{n} \epsilon+a_{n} b_{n} c_{n} \epsilon+a_{n} b_{n} c_{n} \gamma_{n} \epsilon \\
\left.\quad+b_{n}\left\|v_{n}(w)-\xi(w)\right\|+\delta_{n} M(w)\right] & +a_{n} b_{n} \lambda_{n} M(w)+a_{n} b_{n} \beta_{n} \epsilon+a_{n} \delta_{n} M(w) \\
+a_{n} \alpha_{n} \epsilon+\sigma_{n} M(w) & +a_{n} \alpha_{n} \epsilon+\sigma_{n} M(w) \\
=\left(1-a_{n}-\sigma_{n}\right)\left\|\xi_{n}(w)-\xi(w)\right\|+a_{n} \epsilon & =\left(1-\sigma_{n}-a_{n} \delta_{n}-a_{n} b_{n} \lambda_{n}\right)\left\|\xi_{n}(w)-\xi(w)\right\| \\
+a_{n}\left(1-b_{n}-\delta_{n}\right)\left\|\xi_{n}(w)-\xi(w)\right\|+a_{n} b_{n} \epsilon & +\left[a_{n}+a_{n} b_{n}+a_{n} b_{n} c_{n}+a_{n} b_{n} c_{n} \gamma_{n}+a_{n} b_{n} \beta_{n}+a_{n} \alpha_{n}\right] \epsilon \\
+a_{n} b_{n}\left\|v_{n}(w)-\xi(w)\right\| & +\left[a_{n} b_{n} \lambda_{n}+a_{n} \delta_{n}+\sigma_{n}\right] M(w) \\
+a_{n} \delta_{n} M(w)+a_{n} \alpha_{n} \epsilon+\sigma_{n} M(w) & \leq
\end{array}
$$$$
=\left(1-\sigma_{n}-a_{n} b_{n}-a_{n} \delta_{n}\right)\left\|\xi_{n}(w)-\xi(w)\right\|
$$$$
+a_{n} \epsilon+a_{n} b_{n} \epsilon+a_{n} b_{n}\left\|v_{n}(w)-\xi(w)\right\|
$$$$
+a_{n} \delta_{n} M(w)+a_{n} \alpha_{n} \epsilon+\sigma_{n} M(w)
$$$$
\leq\left(1-\sigma_{n}-a_{n} b_{n}-a_{n} \delta_{n}\right)\left\|\xi_{n}(w)-\xi(w)\right\|
$$$$
+a_{n} \epsilon+a_{n} b_{n} \epsilon+a_{n} b_{n}\left[\left\|\zeta_{n}(w)-\xi(w)\right\|+\beta_{n} \epsilon\right]
$$$$
+a_{n} \delta_{n} M(w)+a_{n} \alpha_{n} \epsilon+\sigma_{n} M(w)
$$$$
=\left(1-\sigma_{n}-a_{n} b_{n}-a_{n} \delta_{n}\right)\left\|\xi_{n}(w)-\xi(w)\right\|
$$$$
+a_{n} \epsilon+a_{n} b_{n} \epsilon+a_{n} b_{n}\left\|\zeta_{n}(w)-\xi(w)\right\|
$$$$
+a_{n} b_{n} \beta_{n} \epsilon+a_{n} \delta_{n} M(w)+a_{n} \alpha_{n} \epsilon+\sigma_{n} M(w)
$$$$
\leq\left(1-\sigma_{n}-a_{n} b_{n}-a_{n} \delta_{n}\right)\left\|\xi_{n}(w)-\xi(w)\right\|+a_{n} \epsilon
$$$$
+a_{n} b_{n} \epsilon+a_{n} b_{n}\left(1-c_{n}-\lambda_{n}\right)\left\|\xi_{n}(w)-\xi(w)\right\|
$$$$
+a_{n} b_{n} c_{n} \epsilon+a_{n} b_{n} c_{n}\left\|\tau_{n}(w)-\xi(w)\right\|
$$$$
+a_{n} b_{n} \lambda_{n} M(w)+a_{n} b_{n} \beta_{n} \epsilon+a_{n} \delta_{n} M(w)
$$$$
+a_{n} \alpha_{n} \epsilon+\sigma_{n} M(w)
$$$$
=\left(1-\sigma_{n}-a_{n} \delta_{n}-a_{n} b_{n} c_{n}-a_{n} b_{n} \lambda_{n}\right)
$$$$
\times\left\|\xi_{n}(w)-\xi(w)\right\|+a_{n} \epsilon+a_{n} b_{n} \epsilon
$$$$
+a_{n} b_{n} c_{n} \epsilon+a_{n} b_{n} c_{n}\left\|\tau_{n}(w)-\xi(w)\right\|
$$$$
+a_{n} b_{n} \lambda_{n} M(w)+a_{n} b_{n} \beta_{n} \epsilon+a_{n} \delta_{n} M(w)
$$$$
+a_{n} \alpha_{n} \epsilon+\sigma_{n} M(w)
$$$$
\leq\left(1-\sigma_{n}-a_{n} \delta_{n}-a_{n} b_{n} c_{n}-a_{n} b_{n} \lambda_{n}\right)\left\|\xi_{n}(w)-\xi(w)\right\|
$$$$
+a_{n} \epsilon+a_{n} b_{n} \epsilon+a_{n} b_{n} c_{n} \epsilon+a_{n} b_{n} c_{n}
$$$$
\times\left\|\xi_{n}(w)-\xi(w)\right\|+a_{n} b_{n} c_{n} \gamma_{n} \epsilon+a_{n} b_{n} \lambda_{n} M(w)
$$$$
+a_{n} b_{n} \beta_{n} \epsilon+a_{n} \delta_{n} M(w)+a_{n} \alpha_{n} \epsilon+\sigma_{n} M(w)
$$

Let $R_{n}(w)=6 \epsilon+\left(\lambda_{n}+\delta_{n}+\sigma_{n}\right) M(w)$; then, $\sum_{n=1}^{\infty} R_{n}(w)<\infty$ for all $w \in \Omega$.

$$
\text { It follows by (34) that }
$$

$$
\begin{aligned}
\inf _{\xi(w) \in F} & \left\|\xi_{n+1}(w)-\xi(w)\right\| \\
& \leq \inf _{\xi(w) \in F}\left\|\xi_{n}(w)-\xi(w)\right\|+R_{n}(w), \quad \forall n \geq n_{0}, w \in \Omega .
\end{aligned}
$$

From (35) and $\sum_{n=1}^{\infty} R_{n}(w)<\infty$ for all $w \in \Omega$, we have

$$
d\left(\xi_{n+1}(w), F\right) \leq d\left(\xi_{n}(w), F\right)+R_{n}(w), \quad \forall w \in \Omega .
$$

By Lemma 7 and (36), it follows that $\lim _{n \rightarrow \infty} d\left(\xi_{n}(w), F\right)$ exists for all $\xi(w) \in F=\bigcap_{i=1}^{3} R F\left(T_{i}\right)$ and $w \in \Omega$.

Since $\lim \inf _{n \rightarrow \infty} d\left(\xi_{n}(w), F\right)=0$, then we have

$$
\lim _{n \rightarrow \infty} d\left(\xi_{n}(w), F\right)=0, \quad w \in \Omega \text {. }
$$

Next, we prove that $\xi_{n}(w)$ is a Cauchy sequence in $E$ for each $w \in \Omega$.

For $n \geq n_{0}, m \geq n_{1}$, and $\xi(w) \in F$, we have by (35) that

$$
\begin{aligned}
& \left\|\xi_{n+m}(w)-\xi(w)\right\| \\
& \quad \leq\left\|\xi_{n+m-1}(w)-\xi(w)\right\|+R_{n+m-1}(w) \\
& \quad \leq\left\|\xi_{n+m-2}(w)-\xi(w)\right\|+R_{n+m-1}(w)+R_{n+m-2}(w) \\
& \quad \vdots \\
& \quad \leq\left\|\xi_{n}(w)-\xi(w)\right\|+\sum_{k=n}^{n+m-1} R_{k}(w) .
\end{aligned}
$$

Therefore, by using (38), we have for each $w \in \Omega$,

$$
\begin{aligned}
& \left\|\xi_{n+m}(w)-\xi_{n}(w)\right\| \\
& \quad \leq\left\|\xi_{n+m}(w)-\xi(w)\right\|+\left\|\xi_{n}(w)-\xi(w)\right\| \\
& \quad \leq 2\left\|\xi_{n}(w)-\xi(w)\right\|+\sum_{k=n}^{\infty} R_{k}(w) .
\end{aligned}
$$


Since $\xi(w) \in F$ and by (39), we have for each $w \in \Omega$,

$$
\begin{aligned}
& \left\|\xi_{n+m}(w)-\xi_{n}(w)\right\| \\
& \quad \leq 2 d\left(\xi_{n}(w), F\right)+\sum_{k=n}^{\infty} R_{k}(w), \quad \forall n \geq n_{0} .
\end{aligned}
$$

Since $\lim _{n \rightarrow \infty} d\left(\xi_{n}(w), F\right)=0$ and $\sum_{n=1}^{\infty} R_{n}(w)<\infty$, for given $\epsilon>0$, there exists a positive integer $n_{1} \geq n_{0}$ such that $d\left(\xi_{n}(w), F\right)<\epsilon / 4$ and $\sum_{n=1}^{\infty} R_{n}(w)<\epsilon / 2$. We have

$$
\left\|\xi_{n+m}(w)-\xi_{n}(w)\right\|<\epsilon, \quad \forall w \in \Omega
$$

or

$$
\lim _{n \rightarrow \infty}\left\|\xi_{n+m}(w)-\xi_{n}(w)\right\|=0, \quad \forall w \in \Omega
$$

this shows that $\xi_{n}(w)$ is a Cauchy sequence in $C$ for each $w \in$ $\Omega$.

Since $E$ is complete and $C$ is a closed subset of $E$ and so it is complete, then there exists a $p(w) \in C$ such that $\xi_{n}(w) \rightarrow$ $p(w)$ as $n \rightarrow \infty$, for all $w \in \Omega$.

Now, we show that $p(w) \in F$.

By contradiction, we assume that $p(w)$ does not belong to $F$. Since $F$ is closed set, $d(p(w), F)>0$. By using the fact that $\lim _{n \rightarrow \infty} d\left(\xi_{n}(w), F\right)=0$, it follows that for all $\xi(w) \in F$,

$$
\begin{aligned}
& \|p(w)-\xi(w)\| \\
& \quad \leq\left\|p(w)-\xi_{n}(w)\right\|+\left\|\xi_{n}(w)-\xi(w)\right\| .
\end{aligned}
$$

This implies that

$$
\begin{aligned}
d(p(w), F) & \leq\left\|p(w)-\xi_{n}(w)\right\|+d\left(\xi_{n}(w), F\right) \\
& \leq 0(\text { as } n \rightarrow \infty)
\end{aligned}
$$

which is a contradiction. Hence, $p(w) \in F$.

Corollary 9. Suppose that the conditions in Theorem 8 are satisfied. Then the random iterative sequence $\xi_{n}(w)$ generated by (17) converges to a common random fixed point $\xi(w)$ if and only if for all $w \in \Omega$, there exists a subsequence $\xi_{n_{j}}(w)$ of $\xi_{n}(w)$ which converges to $\xi(w)$.

Theorem 10. Let $E$ be a real separable Banach space and $C a$ nonempty closed convex subset of $E$ with $P$ as a nonexpansive retraction. Let $T_{i}: \Omega \times C \rightarrow E, i=1,2,3$, be three asymptotically quasi-nonexpansive nonself random mappings with $F=\bigcap_{i=1}^{3} R F\left(T_{i}\right) \neq \phi$, for all $w \in \Omega$. Suppose that $\left\{\xi_{n}(w)\right\},\left\{\eta_{n}(w)\right\}$, and $\left\{\zeta_{n}(w)\right\}$ are the sequences defined as in (17) where $\left\{a_{n}\right\},\left\{b_{n}\right\},\left\{c_{n}\right\},\left\{\alpha_{n}\right\},\left\{\beta_{n}\right\},\left\{\gamma_{n}\right\},\left\{\sigma_{n}\right\},\left\{\delta_{n}\right\}$, and $\left\{\lambda_{n}\right\}$ are sequences in $[0,1]$ such that $a_{n}+\sigma_{n} \leq 1, b_{n}+$ $\delta_{n} \leq 1, c_{n}+\lambda_{n} \leq 1$, and $\left\{f_{n}(w)\right\},\left\{g_{n}(w)\right\},\left\{h_{n}(w)\right\}$ are bounded sequences of measurable functions from $\Omega$ to $C$ with the following restrictions: $\sum_{n=1}^{\infty} \sigma_{n}<\infty, \sum_{n=1}^{\infty} \delta_{n}<\infty$ and $\sum_{n=1}^{\infty} \lambda_{n}<\infty$. Then, $\left\{\xi_{n}(w)\right\}$ converge to a common random fixed point of $T_{1}, T_{2}$, and $T_{3}$ if and only if

$$
\liminf _{n \rightarrow \infty} d\left(\xi_{n}(w), F\right)=0, \quad w \in \Omega
$$

Proof. Since $T_{i}: \Omega \times C \rightarrow E, i=1,2,3$, are three asymptotically quasi-nonexpansive nonself random mappings, by Remark 2, they are asymptotically quasi-nonexpansive-type nonself random mappings the conclusion of Theorem 10 can be proved from Theorem 8 immediately.

Theorem 11. Let $E$ be a real separable Banach space and $C$ be a nonempty closed convex subset of $E$ with $P$ as a nonexpansive retraction. Let $T_{i}: \Omega \times C \rightarrow E, i=1,2,3$, be three asymptotically nonexpansive nonself random mappings with $F=\bigcap_{i=1}^{3} R F\left(T_{i}\right) \neq \phi$, for all $w \in \Omega$. Suppose that $\left\{\xi_{n}(w)\right\},\left\{\eta_{n}(w)\right\}$ and $\left\{\zeta_{n}(w)\right\}$ are the sequences defined as in (17) where $\left\{a_{n}\right\},\left\{b_{n}\right\},\left\{c_{n}\right\},\left\{\alpha_{n}\right\},\left\{\beta_{n}\right\},\left\{\gamma_{n}\right\},\left\{\sigma_{n}\right\},\left\{\delta_{n}\right\}$, and $\left\{\lambda_{n}\right\}$ are sequences in $[0,1]$ such that $a_{n}+\sigma_{n} \leq 1, b_{n}+$ $\delta_{n} \leq 1, c_{n}+\lambda_{n} \leq 1$, and $\left\{f_{n}(w)\right\},\left\{g_{n}(w)\right\},\left\{h_{n}(w)\right\}$ are bounded sequences of measurable functions from $\Omega$ to $C$ with the following restrictions: $\sum_{n=1}^{\infty} \sigma_{n}<\infty, \sum_{n=1}^{\infty} \delta_{n}<\infty$, and $\sum_{n=1}^{\infty} \lambda_{n}<\infty$. Then, $\left\{\xi_{n}(w)\right\}$ converge to a common random fixed point of $T_{1}, T_{2}$, and $T_{3}$ if and only if

$$
\liminf _{n \rightarrow \infty} d\left(\xi_{n}(w), F\right)=0, \quad w \in \Omega .
$$

Proof. Since $T_{i}: \Omega \times C \rightarrow E, i=1,2,3$, are three asymptotically nonexpansive nonself random mappings, by Remark 2, they are asymptotically nonexpansive-type nonself random mappings, and therefore they are asymptotically quasi-nonexpansive-type nonself random mappings; the conclusion of Theorem 11 can be obtained from Theorem 8 immediately.

Now, we can extend and generalize Theorems 8,10 , and 11 by using random iterative scheme (18) as follows.

Theorem 12. Let $E$ be a real separable Banach space and $C a$ nonempty closed convex subset of $E$ with $P$ as a nonexpansive retraction. Let $T_{i}: \Omega \times C \rightarrow E, i=1,2, \ldots, N$, be $N$ asymptotically quasi-nonexpansive-type nonself random mappings with $F=\bigcap_{i=1}^{N} R F\left(T_{i}\right) \neq \phi$, for all $w \in \Omega$. Suppose that $\left\{\xi_{n}(w)\right\}$ is the sequence defined as in (18) where $\left\{a_{n}^{(i)}\right\},\left\{\alpha_{n}^{(i)}\right\}$, and $\left\{\sigma_{n}^{(i)}\right\} \quad(i=1,2, \ldots, N)$ are sequences in $[0,1]$ such that $a_{n}^{(i)}+\sigma_{n}^{(i)} \leq 1$ for all $i=1,2, \ldots, N$ and $\left\{f_{n}^{(i)}(w)\right\} \quad(i=$ $1,2, \ldots, N)$ are bounded sequences of measurable functions from $\Omega$ to $C$ with the following restrictions: $\sum_{n=1}^{\infty} \sigma_{n}^{(i)}<\infty$, for all $(i=1,2, \ldots, N)$. Then $\left\{\xi_{n}(w)\right\}$ converge to a common random fixed point of $T_{1}, T_{2}, \ldots, T_{N}$ if and only if

$$
\liminf _{n \rightarrow \infty} d\left(\xi_{n}(w), F\right)=0, \quad w \in \Omega
$$

Theorem 13. Let $E$ be a real separable Banach space and $C$ be a nonempty closed convex subset of $E$ with $P$ as a nonexpansive retraction. Let $T_{i}: \Omega \times C \rightarrow E, i=1,2, \ldots, N$ be $N$ asymptotically quasi-nonexpansive nonself random mappings with $F=\bigcap_{i=1}^{N} R F\left(T_{i}\right) \neq \phi$, for all $w \in \Omega$. Suppose that $\left\{\xi_{n}(w)\right\}$ be the sequence defined as in (18) where $\left\{a_{n}^{(i)}\right\},\left\{\alpha_{n}^{(i)}\right\}$, and $\left\{\sigma_{n}^{(i)}\right\},(i=1,2, \ldots, N)$ are sequences in $[0,1]$ such that $a_{n}^{(i)}+\sigma_{n}^{(i)} \leq 1$ for all $i=1,2, \ldots, N$ and $\left\{f_{n}^{(i)}(w)\right\}, \quad(i=$ $1,2, \ldots, N)$ are bounded sequences of measurable functions from $\Omega$ to $C$ with the following restrictions: $\sum_{n=1}^{\infty} \sigma_{n}^{(i)}<\infty$ 
for all $(i=1,2, \ldots, N)$. Then $\left\{\xi_{n}(w)\right\}$ converge to a common random fixed point of $T_{1}, T_{2}, \ldots, T_{N}$ if and only if

$$
\liminf _{n \rightarrow \infty} d\left(\xi_{n}(w), F\right)=0, \quad w \in \Omega
$$

Theorem 14. Let $E$ be a real separable Banach space and $C$ be a nonempty closed convex subset of $E$ with $P$ as a nonexpansive retraction. Let $T_{i}: \Omega \times C \rightarrow E, i=1,2, \ldots, N$ be $N$ asymptotically nonexpansive nonself random mappings with $F=\bigcap_{i=1}^{N} R F\left(T_{i}\right) \neq \phi$, for all $w \in \Omega$. Suppose that $\left\{\xi_{n}(w)\right\}$ is the sequence defined as in (18) where $\left\{a_{n}^{(i)}\right\},\left\{\alpha_{n}^{(i)}\right\}$, and $\left\{\sigma_{n}^{(i)}\right\} \quad(i=$ $1,2, \ldots, N)$ are sequences in $[0,1]$ such that $a_{n}^{(i)}+\sigma_{n}^{(i)} \leq 1$ for all $i=1,2, \ldots, N$ and $\left\{f_{n}^{(i)}(w)\right\} \quad(i=1,2, \ldots, N)$ are bounded sequences of measurable functions from $\Omega$ to $C$ with the following restrictions $\sum_{n=1}^{\infty} \sigma_{n}^{(i)}<\infty$ for all $(i=1,2, \ldots, N)$. Then, $\left\{\xi_{n}(w)\right\}$ converge to a common random fixed point of $T_{1}, T_{2}, \ldots, T_{N}$ if and only if

$$
\liminf _{n \rightarrow \infty} d\left(\xi_{n}(w), F\right)=0, \quad w \in \Omega .
$$

Senter and Dotson [40] defined Condition (A) as follows.

Definition 15 (see [40]). A mapping $T: C \rightarrow C$ where $C$ is a subset of a Banach space $E$ with $F(T) \neq \phi$ is said to satisfy Condition (A) if there exists a nondecreasing function $f:[0, \infty) \rightarrow[0, \infty)$ with $f(0)=0, f(r)>0$, for all $r \in(0, \infty)$ such that for all $x \in C$,

$$
\|x-T x\| \geq f(d(x, F(T))),
$$

where $d(x, F(T))=\inf \{\|x-p\|: p \in F(T)\}$.

As an application, we can apply Theorem 8 and Condition (A) to obtain a convergences theorem for scheme (17).

Theorem 16. Let $E$ be a real uniformly separable Banach space and $C$ a nonempty closed convex subset of $E$ with $P$ as a nonexpansive retraction. Let $T_{i}: \Omega \times C \rightarrow E, i=1,2,3$, be three asymptotically quasi-nonexpansive-type nonself random mappings with $F=\bigcap_{i=1}^{3} R F\left(T_{i}\right) \neq \phi$, for all $w \in \Omega$. Suppose that $\left\{\xi_{n}(w)\right\},\left\{\eta_{n}(w)\right\}$ and $\left\{\zeta_{n}(w)\right\}$ are the sequences defined as in (17) where $\left\{a_{n}\right\},\left\{b_{n}\right\},\left\{c_{n}\right\},\left\{\alpha_{n}\right\},\left\{\beta_{n}\right\},\left\{\gamma_{n}\right\},\left\{\sigma_{n}\right\},\left\{\delta_{n}\right\}$, and $\left\{\lambda_{n}\right\}$ are sequences in $[0,1]$ such that $a_{n}+\sigma_{n} \leq 1, b_{n}+$ $\delta_{n} \leq 1, c_{n}+\lambda_{n} \leq 1$ and $\left\{f_{n}(w)\right\},\left\{g_{n}(w)\right\},\left\{h_{n}(w)\right\}$ are bounded sequences of measurable functions from $\Omega$ to $C$ with the following restrictions: $\sum_{n=1}^{\infty} \sigma_{n}<\infty, \sum_{n=1}^{\infty} \delta_{n}<\infty$, and $\sum_{n=1}^{\infty} \lambda_{n}<\infty$. Suppose one of the mappings $T_{i}, i=$ $1,2,3$, satisfying Condition (A) and the following condition: $\lim _{n \rightarrow \infty}\left\|\xi_{n}(w)-T\left(w, \xi_{n}(w)\right)\right\|=0$, for all $w \in \Omega$. Then, $\left\{\xi_{n}(w)\right\}$ converge to a common random fixed point of $T_{1}, T_{2}$, and $T_{3}$.

Proof. From Theorem 8, we have $\lim _{n \rightarrow \infty}\left\|\xi_{n}(w)-\xi(w)\right\|$, and $\lim _{n \rightarrow \infty} d\left(\xi_{n}(w), F\right)$ exists. Let one of the mappings $T_{i}$, say $T_{1}$ satisfy Condition $(\mathrm{A})$ and $\lim _{n \rightarrow \infty} \| \xi_{n}(w)-$ $T_{1}\left(w, \xi_{n}(w)\right) \|=0$; then, we have for all $w \in \Omega$,

$$
\lim _{n \rightarrow \infty} f\left(d\left(\xi_{n}(w), F\right)\right) \leq \lim _{n \rightarrow \infty}\left\|\xi_{n}(w)-T_{1}\left(w, \xi_{n}(w)\right)\right\|=0 .
$$

By the property of $f$ and since $\lim _{n \rightarrow \infty} d\left(\xi_{n}(w), F\right)$ exists, we have that

$$
\lim _{n \rightarrow \infty} d\left(\xi_{n}(w), F\right)=0
$$

By Theorem $8,\left\{\xi_{n}(w)\right\}$ converge to a common random fixed point of $T_{1}, T_{2}$, and $T_{3}$.

\section{Acknowledgment}

The authors would like to extend their sincerest thanks to the anonymous referees and editors for the exceptional review of this work. The suggestions and recommendations in the report increased the quality of their paper.

\section{References}

[1] O. Hanš, "Reduzierende zufällige Transformationen," Czechoslovak Mathematical Journal, vol. 7, pp. 154-158, 1957.

[2] O. Hanš, "Random operator equations," in Proceeding of the 4th Barkeley Symposium on Mathematical Statistics and Probability, vol. 2, pp. 185-202, University of California Press, Berkeley, Calif, USA, 1961

[3] A. Špaček, "Zufällige Gleichungen," Czechoslovak Mathematical Journal, vol. 5, pp. 462-466, 1955.

[4] A. T. Bharucha-Reid, "Fixed point theorems in probabilistic analysis," Bulletin of the American Mathematical Society, vol. 82, no. 5, pp. 641-657, 1976.

[5] I. Beg, "Approximation of random fixed points in normed spaces," Nonlinear Analysis: Theory, Methods \& Applications, vol. 51, no. 8, pp. 1363-1372, 2002.

[6] I. Beg, "Minimal displacement of random variables under Lipschitz random maps," Topological Methods in Nonlinear Analysis, vol. 19, no. 2, pp. 391-397, 2002.

[7] I. Beg and N. Shahzad, "Random fixed point theorems for nonexpansive and contractive-type random operators on Banach spaces," Journal of Applied Mathematics and Stochastic Analysis, vol. 7, no. 4, pp. 569-580, 1994.

[8] S. Itoh, "Random fixed-point theorems with an application to random differential equations in Banach spaces," Journal of Mathematical Analysis and Applications, vol. 67, no. 2, pp. 261273, 1979.

[9] N. S. Papageorgiou, "Random fixed point theorems for measurable multifunctions in Banach spaces," Proceedings of the American Mathematical Society, vol. 97, no. 3, pp. 507-514, 1986.

[10] H. K. Xu, "Some random fixed point theorems for condensing and nonexpansive operators," Proceedings of the American Mathematical Society, vol. 110, no. 2, pp. 395-400, 1990.

[11] K. Goebel and W. A. Kirk, "A fixed point theorem for asymptotically nonexpansive mappings," Proceedings of the American Mathematical Society, vol. 35, pp. 171-174, 1972.

[12] J. Gornicki, "Weak convergence theorems for asymptotically nonexpansive mappings in uniformly convex Banach spaces," Commentationes Mathematicae Universitatis Carolinae, vol. 30, no. 2, pp. 249-252, 1989.

[13] M. O. Osilike and S. C. Aniagbosor, "Weak and strong convergence theorems for fixed points of asymptotically nonexpansive mappings," Mathematical and Computer Modelling, vol. 32, no. 10, pp. 1181-1191, 2000. 
[14] J. Schu, "Iterative construction of fixed points of asymptotically nonexpansive mappings," Journal of Mathematical Analysis and Applications, vol. 158, no. 2, pp. 407-413, 1991.

[15] J. Schu, "Weak and strong convergence to fixed points of asymptotically nonexpansive mappings," Bulletin of the Australian Mathematical Society, vol. 43, no. 1, pp. 153-159, 1991.

[16] W. V. Petryshyn and T. E. Williamson, Jr., "Strong and weak convergence of the sequence of successive approximations for quasi-nonexpansive mappings," Journal of Mathematical Analysis and Applications, vol. 43, pp. 459-497, 1973.

[17] M. K. Ghosh and L. Debnath, "Convergence of Ishikawa iterates of quasi-nonexpansive mappings," Journal of Mathematical Analysis and Applications, vol. 207, no. 1, pp. 96-103, 1997.

[18] Q. H. Liu, "Iterative sequences for asymptotically quasinonexpansive mappings," Journal of Mathematical Analysis and Applications, vol. 259, no. 1, pp. 1-7, 2001.

[19] Q. H. Liu, "Iterative sequences for asymptotically quasinonexpansive mappings with error member," Journal of Mathematical Analysis and Applications, vol. 259, no. 1, pp. 18-24, 2001.

[20] M. A. Noor, "New approximation schemes for general variational inequalities," Journal of Mathematical Analysis and Applications, vol. 251, no. 1, pp. 217-229, 2000.

[21] B. Xu and M. A. Noor, "Fixed-point iterations for asymptotically nonexpansive mappings in Banach spaces," Journal of Mathematical Analysis and Applications, vol. 267, no. 2, pp. 444-453, 2002.

[22] S. Suantai, "Weak and strong convergence criteria of Noor iterations for asymptotically nonexpansive mappings," Journal of Mathematical Analysis and Applications, vol. 311, no. 2, pp. 506-517, 2005.

[23] J. S. Jung and S. S. Kim, "Strong convergence theorems for nonexpansive nonself-mappings in Banach spaces," Nonlinear Analysis: Theory, Methods \& Applications, vol. 33, no. 3, pp. 321329, 1998.

[24] S. Y. Matsushita and D. Kuroiwa, "Strong convergence of averaging iterations of nonexpansive nonself-mappings," Journal of Mathematical Analysis and Applications, vol. 294, no. 1, pp. 206214, 2004.

[25] N. Shahzad, "Approximating fixed points of non-self nonexpansive mappings in Banach spaces," Nonlinear Analysis: Theory, Methods \& Applications, vol. 61, no. 6, pp. 1031-1039, 2005.

[26] W. Takahashi and G. E. Kim, "Strong convergence of approximants to fixed points of nonexpansive nonself-mappings in Banach spaces," Nonlinear Analysis: Theory, Methods \& Applications, vol. 32, no. 3, pp. 447-454, 1998.

[27] H. K. Xu and X. M. Yin, "Strong convergence theorems for nonexpansive non-self-mappings," Nonlinear Analysis: Theory, Methods \& Applications, vol. 24, no. 2, pp. 223-228, 1995.

[28] C. E. Chidume, E. U. Ofoedu, and H. Zegeye, "Strong and weak convergence theorems for asymptotically nonexpansive mappings," Journal of Mathematical Analysis and Applications, vol. 280, no. 2, pp. 364-374, 2003.

[29] L. Wang, "Strong and weak convergence theorems for common fixed point of nonself asymptotically nonexpansive mappings," Journal of Mathematical Analysis and Applications, vol. 323, no. 1, pp. 550-557, 2006.

[30] I. Beg and M. Abbas, "Iterative procedures for solutions of random operator equations in Banach spaces," Journal of Mathematical Analysis and Applications, vol. 315, no. 1, pp. 181-201, 2006.
[31] Y. X. Tian, S. S. Chang, and J. L. Huang, "On the approximation problem of common fixed points for a finite family of non-self asymptotically quasi-nonexpansive-type mappings in Banach spaces," Computers \& Mathematics with Applications, vol. 53, no. 12, pp. 1847-1853, 2007.

[32] S. Thianwan, "Weak and strong convergence theorems for new iterations with errors for nonexpansive nonself-mapping," Thai Journal of Mathematics, vol. 6, no. 3, pp. 27-38, 2008.

[33] H. Kiziltunc, "On common fixed points of a new iteration for two nonself asymptotically quasi-nonexpansive-type mappings in Banach spaces," Journal of Nonlinear Analysis and Optimization, vol. 2, no. 2, pp. 259-267, 2011.

[34] W. Q. Deng, L. Wang, and Y. J. Chen, "Strong and weak convergence theorems for common fixed points of two nonself asymptotically nonexpansive mappings in Banach spaces," International Mathematical Forum, vol. 7, no. 9-12, pp. 407-417, 2012.

[35] S. Plubtieng, P. Kumam, and R. Wangkeeree, "Random threestep iteration scheme and common random fixed point of three operators," Journal of Applied Mathematics and Stochastic Analysis, vol. 2007, Article ID 82517, 10 pages, 2007.

[36] S. Plubtieng, R. Wangkeeree, and R. Punpaeng, "On the convergence of modified Noor iterations with errors for asymptotically nonexpansive mappings," Journal of Mathematical Analysis and Applications, vol. 322, no. 2, pp. 1018-1029, 2006.

[37] X. W. Zhou and L. Wang, "Approximation of random fixed points of non-self asymptotically nonexpansive random mappings," International Mathematical Forum, vol. 2, no. 37-40, pp. 1859-1868, 2007.

[38] G. S. Saluja, "Approximation of common random fixed point for a finite family of non-self asymptotically nonexpansive random mappings," Demonstratio Mathematica, vol. 42, no. 3, pp. 581598, 2009.

[39] K. K. Tan and H. K. Xu, "Approximating fixed points of nonexpansive mappings by the Ishikawa iteration process," Journal of Mathematical Analysis and Applications, vol. 178, no. 2, pp. 301308, 1993.

[40] H. F. Senter and W. G. Dotson Jr., "Approximating fixed points of nonexpansive mappings," Proceedings of the American Mathematical Society, vol. 44, pp. 375-380, 1974. 


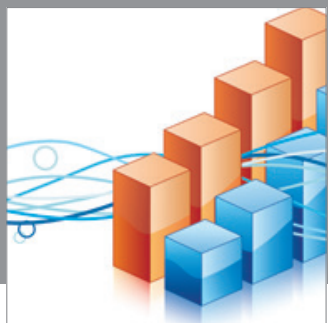

Advances in

Operations Research

mansans

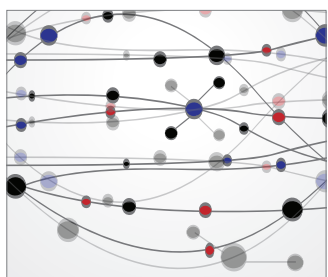

The Scientific World Journal
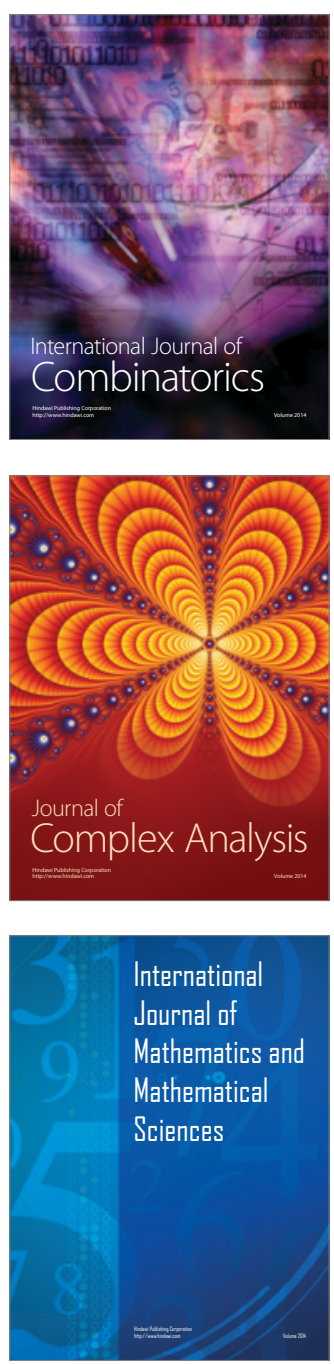
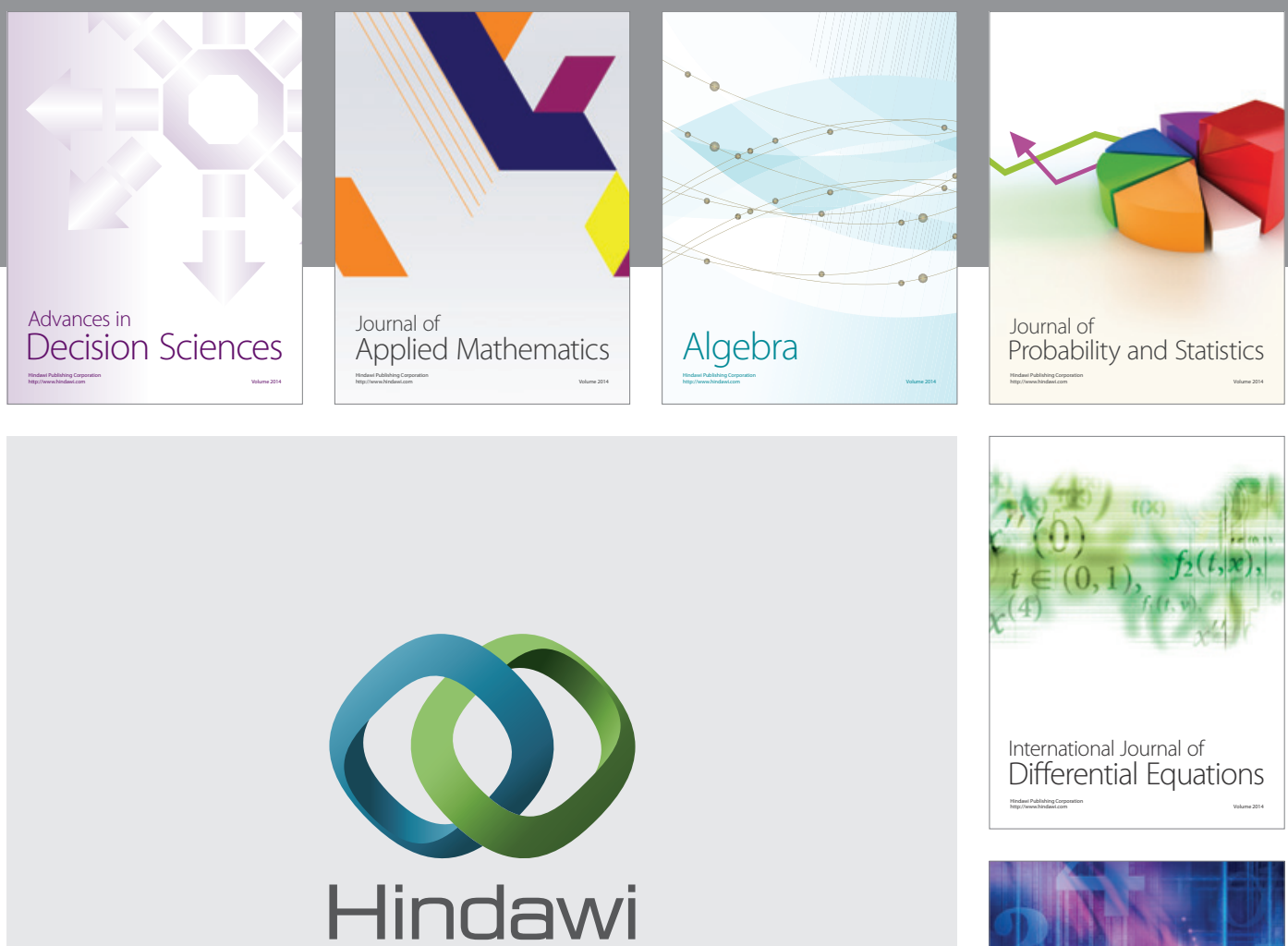

Submit your manuscripts at http://www.hindawi.com
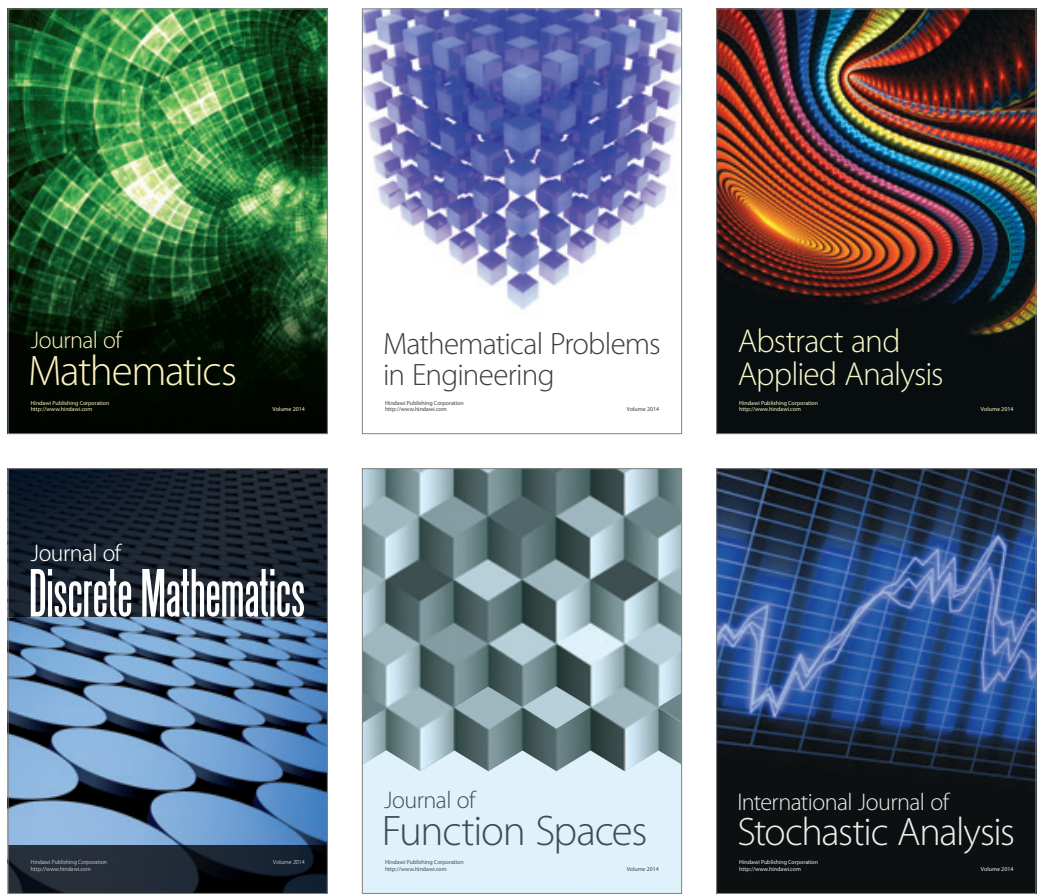

Journal of

Function Spaces

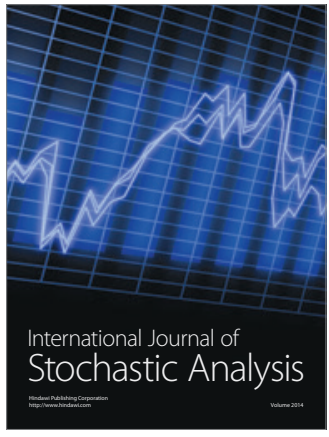

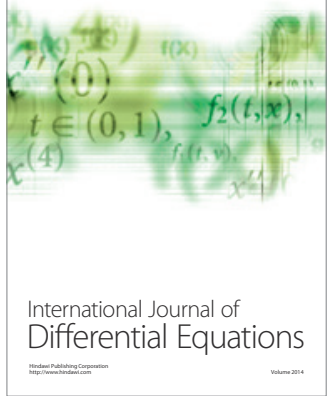
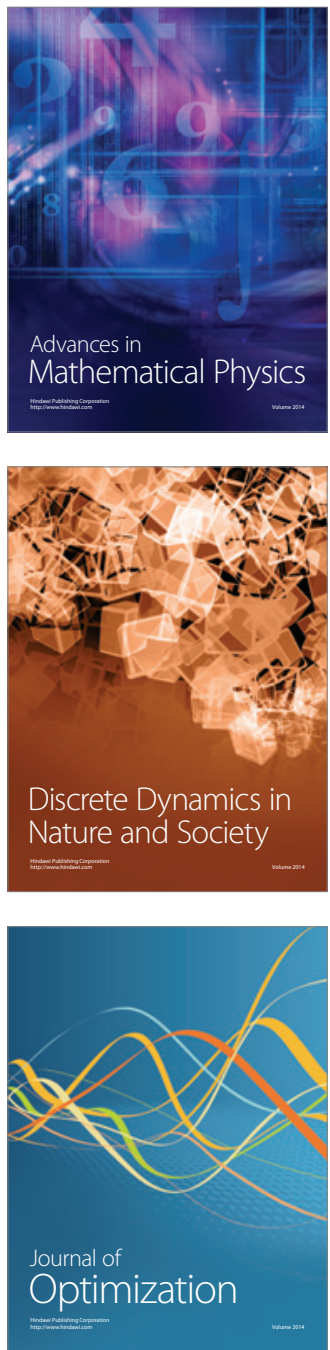